\title{
A Look at Ligand Binding Thermodynamics in Drug Discovery
}

Rafael Claveria-Gimeno ${ }^{1,2,3}$, Sonia Vega ${ }^{1}$, Olga Abian ${ }^{1,2,3,4,5}$, Adrian VelazquezCampoy ${ }^{1,3,4,6 *}$

${ }^{1}$ Institute of Biocomputation and Physics of Complex Systems (BIFI), IQFR-CSIC-BIFI and GBsC-CSIC-BIFI Joint Units, Universidad de Zaragoza, Mariano Esquillor s/n, Edificio I+D, Campus Rio Ebro, Zaragoza, 50018, Spain

${ }^{2}$ Instituto Aragonés de Ciencias de la Salud (IACS), Avda. San Juan Bosco 13, Zaragoza, 50009, Spain

${ }^{3}$ Aragon Institute for Health Research (IIS Aragon), Avda. San Juan Bosco 13, Zaragoza, 50009, Spain

${ }^{4}$ Department of Biochemistry and Molecular and Cell Biology, Universidad de Zaragoza, Pedro Cerbuna 12, Zaragoza, 50009, Spain

${ }^{5}$ Centro de Investigación Biomédica en Red en el Área Temática de Enfermedades Hepáticas y Digestivas (CIBERehd), Barcelona, Spain

${ }^{6}$ Fundación ARAID, Government of Aragon, Maria de Luna 11, Edificio CEEI, Zaragoza, 50018, Spain

*To whom all editorial correspondence should be addressed:

Adrian Velazquez-Campoy

Tel: +34 976762996;

Fax: +34 976762990;

E-mail: adrianvc@unizar.es 
Introduction: Drug discovery is a challenging endeavor requiring the interplay of many different research areas. Gathering information on ligand binding thermodynamics may help considerably in reducing the risk within a high uncertainty scenario, allowing early rejection of flawed compounds and pushing forward optimal candidates. In particular, the free energy, the enthalpy, and the entropy of binding provide fundamental information on the intermolecular forces driving such interaction.

Areas covered: The authors review the current status and recent developments in the application of ligand binding thermodynamics in drug discovery. The thermodynamic binding profile (Gibbs energy, enthalpy, and entropy of binding) can be used for lead selection and optimization (binding enthalpy, selectivity, and adaptability).

Expert opinion: Binding thermodynamics provides fundamental information on the forces driving the formation of the drug-target complex. It has been widely accepted that binding thermodynamics may be used as a decision criterion along the ligand optimization process in drug discovery and development. In particular, the binding enthalpy may be used as a guide when selecting and optimizing compounds over a set of potential candidates. However, this has been recently called into question by arguing certain difficulties and in the light of certain experimental examples.

Keywords: binding adaptability, binding affinity, binding selectivity, enthalpy, entropy, Gibbs energy, isothermal titration calorimetry, ligand binding, ligand optimization, lipophilic efficiency, thermodynamics 


\section{Introduction}

The usual work-flow in drug discovery starts from either computational or experimental high-throughput screening programs, and progressing promising compounds from in vitro testing to assessing ADMET (absorption, distribution, metabolism, excretion and toxicity) properties and efficacy in preclinical animal models. The final goal in drug discovery is the identification of a chemical compound that, by interacting with a certain target, triggers a physiological response that results in the reversion of a pathological scenario caused by an aberrant activity of the target. The chemical compound must fulfil rigorous constraints: good pharmacokinetic properties (suitable distribution through the body, as well as appropriate metabolic modification and clearance) and low toxicity (low level of interaction with unwanted secondary targets). Improving binding affinity while improving or maintaining drug-like properties is not an easy task.

According to Paul Ehrlich's statement "corpora non agunt nisi fixata" (a substance is not effective unless it is bound to another), the pharmacological action of a drug relies primarily on its interaction with the key target. Gathering the necessary information through the discovery process on ligand binding, in addition to information on pharmacokinetics and toxicity, and applying stringent filters in a rational way is instrumental for making appropriate decisions regarding ligand optimization. This will considerably reduce the attrition rate in the drug discovery process, allowing early rejection of flawed compounds and pushing forward optimal candidates. In order to increase the rate of success in the hit generation process, as well as in the hit-to-leadto-drug process, preclinical research in target validation and characterization, target selection, and, very importantly, integrated drug discovery strategies are crucial. Those integrated strategies involve applying multiple orthogonal approaches for obtaining valuable information and employing multifaceted decision criteria, where ligand binding thermodynamics may play a key role. Biophysical data, in particular thermodynamic binding information, provide information complementary to biochemical or cellular data, contributing to improve compound prioritization and decision-making in early stages in drug discovery. Importantly, biophysical techniques are key instruments for target characterization, screening design, and hit validation. 
From a basic perspective, the drug-target interaction may be modeled as a simple equilibrium that can be characterized using binding equilibrium parameters and kinetic parameters. Although the kinetic information, epitomized by the recently established concept of residence time [1,2], represents an interesting approach for ligand optimization [3], obtaining precise information on the equilibrium thermodynamics of the interaction is a crucial stage in drug discovery.

Traditionally, the main focus when quantifying binding affinity in drug discovery was set on determining binding parameters such as equilibrium constants (e.g., association, dissociation, and inhibition constants) and operational parameters (e.g., EC50 or IC50). While the former parameters are true equilibrium constants, the latter operational parameters must be taken with caution since they do not represent true equilibrium constants, they are assay-specific and strongly dependent on experimental set-up and conditions, and they can very often lead to misinterpretations and considerable error when used for ranking compound affinities.

Besides binding affinity, the "reduced" set of thermodynamic ligand binding parameters (Gibbs energy, enthalpy, entropy and heat capacity) provides fundamental information on the interaction event and the intermolecular forces driving such interaction. These binding parameters can be measured directly (by isothermal titration calorimetry) or indirectly (e.g., by spectroscopy, surface plasmon resonance, or differential scanning calorimetry/fluorometry). Because we are interested in high quality information and the direct determination of binding parameters, avoiding if possible the estimation of binding parameters through secondary relationships (e.g., estimation of the binding enthalpy and the binding heat capacity through the van ' $t$ Hoff relationship in spectroscopic techniques), we will restrict this review to isothermal titration calorimetry (ITC). ITC is a technique especially suited for determining experimentally the "reduced" set of thermodynamic ligand binding parameters, as well as the "extended" set of thermodynamic ligand binding parameters (including linkage parameters associated with additional equilibria coupled to the ligand binding) [4-7]. Nowadays, the widespread availability of high-precision ITC instrumentation makes possible including binding thermodynamics into the drug discovery process.

As it will be explained below, the utility of the ligand binding thermodynamic profile (Gibbs energy, enthalpy, and entropy) in the drug discovery and ligand optimization 
process is based on the fact that it reflects and summarizes the interplay between numerous intermolecular interactions implicated in the formation of the drug-target complex: creation of the network of interactions between the drug and target, and rupture of interactions with the solvent, including the contribution of potential conformational and allosteric effects and additional equilibria coupled to the binding. Intermolecular interactions of different nature (hydrogen bonds, van der Waals interactions, ionic pairs, hydrophobic interactions...) contribute differently to the binding affinity and, most importantly, to the binding enthalpy and entropy. Incidentally, all intra- and intermolecular interactions are of electrostatic nature. However, traditionally, some special types of interactions have been named with specific terms: hydrogen bond (a special type of ionic interaction), van der Waals interaction (the interaction between different combinations of instantaneous and permanent dipoles), ionic pair (purely electrostatic interaction between charged groups), hydrophobic interaction (the combination of van der Waals interactions between nonpolar groups and hydrogen bonds between surrounding water molecules), etc. The partition of the binding Gibbs energy into its enthalpic and entropic contributions reflects the mode of interaction between drug and target, i.e., intermolecular interactions responsible for the formation of the drugtarget complex, and molecular-scale phenomena taking place during the complex formation. This reveals an existing link between structural and functional features involved in the formation of the drug-target complex and the energetics of such process.

During lead optimization the binding affinity can be optimized by making the enthalpy of binding more favorable, the entropy of binding more favorable, or by a combination of both. Even though many combinations of enthalpy and entropy values will elicit the same binding affinity (i.e. the same Gibbs energy of binding and the same association constant), the properties and the response of the optimized compounds to changes in the environment or in the protein target are not the same. Besides, as we will discuss below, there are three main factors responsible for improving binding affinity from a thermodynamic point of view: 1) improving ligand-macromolecule interactions over those with the solvent in order to get a more favorable binding enthalpy; 2) making the ligand more hydrophobic in order to get a more favorable solvation entropy; and, 3) pre-shaping the ligand to the geometry of the binding site in order to minimize the 
loss of conformational entropy upon binding. On this basis, it was established that: 1) different optimization strategies based on chemically modifying the drug molecule would affect in a different extent the enthalpy and the entropy of binding (and, consequently, the Gibbs energy of binding); 2) the binding thermodynamic profile or signature of the drug molecule provides information on and conditions its optimization potential; and 3) enthalpic and entropic contributions to the Gibbs energy of binding reflect intermolecular interactions and molecular phenomena of different nature. Consequently, the following hypotheses were made fifteen years ago: 1) an enthalpic optimization (improving the enthalpy of binding) of drug candidates is preferred over an entropic optimization (improving the entropy of binding); and 2) drug candidates with an enthalpically dominated thermodynamic binding profile represent the most promising compounds towards optimization regarding binding affinity, binding selectivity, and susceptibility to mutations in the target [8-11].

Enthalpic optimization relies on a rather simple idea: a large negative binding enthalpy is advantageous regarding binding affinity, target selectivity and ADME properties, because it reflects specific, direct, polar interactions between compound and target; on the other hand, large negative entropic contribution is disadvantageous, because it reflects mainly non-specific desolvation and van der Waals interactions [12-15]. Although different targets have been described to comply with these hypotheses (e.g., HIV-1 protease [11,16], plasmepsin II from P. falciparum [17], HMG-CoA reductase [18], this new paradigm for drug design and optimization based on integrating thermodynamic binding data and implementing an enthalpic optimization is not straightforward, requiring a carefully planned experimental strategy and a close data examination and analysis [19].

Along recent years, it has been realized that reality could be not as simple as it was initially thought: enthalpic and entropic contributions integrate multiple contributions to the ligand-target complex formation (with a dominant role of water molecules), they are extremely sensitive to experimental conditions, and they are affected by enthalpy-entropy compensation $[20,21]$. Therefore, the prospective use of thermodynamics in drug discovery (lead prioritization and optimization) could be somewhat intricate. In fact, it has been called into question very recently because of: 1) its apparent limited applicability to some "well-behaved" protein targets; 2) the 
retrospective nature of the underlying rationale and the apparent need of structural information; 3) a not so straightforward connection between structural/functional and energetic features associated to the drug-target interaction due to the multifactorial character of the enthalpic and the entropic of binding; and 4) the lack of guidance on how to prospectively use the thermodynamic profile in lead generation and optimization programs [22]. Thus, it seems that some of the expectations raised on the prospective application of binding thermodynamics in the selection and optimization of leads, in particular the improved success rate of preclinical drug discovery, have not been met. One of the purposes of this review is to draw the attention into this issue, and show how some weaknesses may be reverted into strengths.

It is obvious that, in principle, studying the binding thermodynamics of the drug-target interaction is an important but limited part of the drug discovery process; many other aspects (basically, those associated with pharmacokinetics and ADME properties) are apparently not related. However, as it will be commented below, valuable information on selectivity, adaptability and potential toxicity of a given drug can be linked to some ligand binding thermodynamics features. Besides, the biophysical techniques employed for providing information on the binding thermodynamics of the drug-target (mainly, ITC, but also many other experimental techniques) can be of help in designing and validating the experimental screening procedure, in establishing target engagement of potential lead compounds and performing secondary screenings, and in assessing selectivity and specificity of the interaction with the target by testing the interaction of lead compounds with potential secondary targets. The selection of a given experimental technique will depend on the information content, its throughput, its requirements for assay development, and its reagent consumption. ITC possesses several important advantages: direct determination of the thermodynamic binding profile in a single experiment, monitoring the interaction in solution, and universality of detection system based on reaction heat with no need for reactant modification. On the other hand, it has some considerable disadvantages: reagent consumption, and low throughput.

Over the past years biophysical techniques have been integrated as key components of drug discovery platforms. The use of biophysical techniques in drug discovery, the current status and the long term expectations, has been reviewed comprehensively 
[23-25]. In this review, we will go first through the foundations of the thermodynamic description of ligand binding. Then, we will discuss: 1) what valuable information can be extracted from the ligand binding thermodynamic profile, 2) how this information can be used within a drug discovery program, and 3) what precautions and caveats must be taken into consideration when including that information within a drug discovery program.

\section{Thermodynamics of ligand binding}

\subsection{The equilibrium constant}

The reversible equilibrium between the free species of reactants, ligand $L$ and macromolecule $\mathrm{M}$, and the macromolecule-ligand complex, $\mathrm{ML}$, is governed by the equilibrium association or dissociation constants, $\mathrm{K}_{\mathrm{a}}$ or $\mathrm{K}_{\mathrm{d}}$ :

$K_{a}=\frac{[M L]}{[M][L]}=\frac{1}{K_{d}}$

Therefore, the ratio between the ligand-bound macromolecule and the ligand-free macromolecule and the ratio between the ligand-bound macromolecule and the total macromolecule are determined by the association constant and the concentration of ligand:

$$
\begin{aligned}
\frac{[M L]}{[M]} & =K_{a}[L] \\
\frac{[M L]}{[M]_{T}} & =\frac{K_{a}[L]}{1+K_{a}[L]}
\end{aligned}
$$

The equilibrium association constant is directly connected to the Gibbs energy of binding, the main thermodynamic potential at constant temperature and pressure:

$\Delta G=-R T \ln K_{a}$

where $\mathrm{R}$ is the ideal gas constant, and $\mathrm{T}$ is the absolute temperature. The required superscript "o" indicating standard conditions ( $1 \mathrm{~mol} / \mathrm{L}, 298.15 \mathrm{~K}, 1 \mathrm{~atm}$ ) has been omitted in Equation 3 and subsequent ones for the sake of clarity.

Additional thermodynamic potentials, the enthalpy and the entropy of binding, derive directly from the equilibrium constant or the Gibbs energy by introducing the temperature derivative: 


$$
\begin{gathered}
\Delta H=-T^{2}\left(\frac{\partial \Delta G / T}{\partial T}\right)_{P, \ldots}=R T^{2}\left(\frac{\partial \ln K_{a}}{\partial T}\right)_{P, \ldots} \\
\Delta S=-\left(\frac{\partial \Delta G}{\partial T}\right)_{P, \ldots}=R \ln K_{a}+R T\left(\frac{\partial \ln K_{a}}{\partial T}\right)_{P, \ldots}
\end{gathered}
$$

which are the two additive terms making up the Gibbs energy:

$\Delta G=\Delta H-T \Delta S$

These equations provide the formalism for determining the binding parameters $(\Delta G$, $\Delta \mathrm{H}$ and $\Delta \mathrm{S}$ ) using spectroscopic techniques by performing titrations at different temperatures. Fortunately, because ITC measures directly the reaction heat, it is the only technique that allows determining with high precision the three binding parameters from a single experiment. Moreover, contrary to non-calorimetric techniques, it is possible to estimate the heat capacity of binding, $\Delta C_{p}$, with reasonable precision by performing ITC experiments at different temperatures:

$\Delta C_{P}=\left(\frac{\partial \Delta H}{\partial T}\right)_{P, \ldots}=T\left(\frac{\partial \Delta S}{\partial T}\right)_{P, \ldots}$

from the slope of the enthalpy vs. temperature plot.

When describing and comparing ligand binding profiles it is convenient to consider the entropic contribution to the Gibbs energy (-T $\Delta S)$, instead of the entropy of binding $(\Delta S)$. The reason behind that is to compare Gibbs energy, enthalpy and entropy on a common basis: with the same units $(\mathrm{kcal} / \mathrm{mol})$ and with the same criterion regarding spontaneity of the thermodynamic process (negative enthalpy and entropy contributions contribute favorably to the binding, while positive contributions oppose the binding). Thus, from now on whenever we refer to the entropy, we are actually referring to the "entropic contribution" (-T $\Delta S)$.

If the ligand binding equilibrium is coupled to a conformational equilibrium, the apparent (or observed) association constant for the binding, $\mathrm{K}_{\mathrm{a}}{ }^{\mathrm{app}}$, is given by:

$K_{a}^{a p p}=\frac{K_{a}}{1+K}$

where $\mathrm{K}$ is the equilibrium constant corresponding to the conformational equilibrium between a non-binding competent conformation $\mathrm{M}^{*}$ and the binding competent conformation $M$ (i.e., $K=\left[\mathrm{M}^{*}\right] /[\mathrm{M}]$ ). The apparent (or observed) binding parameters $\left(\Delta G^{\text {app }}, \Delta H^{\text {app }}, \Delta S^{\text {app }}\right.$ and $\left.\Delta C_{P}^{\text {app }}\right)$ can be calculated applying Equations 3-6 [26-28].

On the other hand, if the ligand binding equilibrium is coupled to another binding equilibrium (that is, there is another ligand, $X$, binding to the macromolecule, and the 
two ligands, $L$ and $X$, bind in a cooperative, negative or positive, fashion), the apparent association constant for the binding, $\mathrm{K}_{\mathrm{a}}{ }^{\mathrm{app}}$ is given by:

$K_{a}^{a p p}=K_{a} \frac{\prod_{j}^{m}\left(1+K_{j}^{\prime}[X]\right)}{\prod_{j}^{m}\left(1+K_{j}[X]\right)}$

where $m$ is the number of binding sites for ligand $X, K_{j}$ is the association constant for ligand $\mathrm{X}$ to binding site $\mathrm{j}$ when ligand $\mathrm{L}$ is not bound to the macromolecule, and $\mathrm{K}_{\mathrm{j}}$ is the association constant for ligand $X$ to binding site $j$ when ligand $L$ is bound. The apparent binding parameters $\left(\Delta \mathrm{G}^{\mathrm{app}}, \Delta \mathrm{H}^{\mathrm{app}}, \Delta \mathrm{S}^{\mathrm{app}}\right.$ and $\left.\Delta \mathrm{C}_{\mathrm{P}}^{\text {app }}\right)$ can be calculated applying Equations 3-6 $[26,27,29]$. If $\mathrm{K}_{\mathrm{j}}^{\prime}=\mathrm{K}_{\mathrm{j}}$ for every $\mathrm{j}$, there is no cooperativity between the binding of both ligands (ligand $L$ binding is not influenced by ligand $X$ binding) and the apparent binding parameters are equal to the intrinsic binding parameters; otherwise, there is cooperativity between the binding of both ligands (ligand $\mathrm{L}$ binding is influenced by ligand $\mathrm{X}$ binding) and the apparent binding parameters are different from the intrinsic binding parameters and contain contributions from ligand $\mathrm{X}$ binding parameters. The situation represented by Equation 8 is very common. For example, $\mathrm{X}$ may represent protons that associate or dissociate from ionizable groups ( $m$ sites located in the macromolecule or in the ligand) depending on whether $\mathrm{L}$ binds or not to the protein; in that case, the apparent association constant (and the apparent binding parameters) for ligand $L$ exhibits a dependency on the $\mathrm{pH}$ (that is, the free concentration of ligand $\mathrm{X}$ ):

$K_{a}^{a p p}=K_{a} \frac{\Pi_{j}^{m}\left(1+10^{p K_{a, j}^{\prime}-p H}\right)}{\prod_{j}^{m}\left(1+10^{p K_{a, j}-p H}\right)}$

where $\mathrm{pK}_{a, j}$ is the acid dissociation constant for the protonation site $\mathrm{j}$ when ligand $\mathrm{L}$ is not bound to the macromolecule, and $\mathrm{pK}^{\prime}{ }_{\mathrm{a}, \mathrm{j}}$ is the acid dissociation constant for the protonation site $\mathrm{j}$ when ligand $\mathrm{L}$ is bound. As another example, $\mathrm{X}$ may represent $\mathrm{a}$ competitive ligand for ligand $L$ binding to the same site in the macromolecule; in that case, the apparent association constant (and the apparent binding parameters) for ligand L exhibits a dependency on the concentration of ligand X:

$K_{a}^{a p p}=K_{a} \frac{1}{1+K_{X}[X]}$

where $K_{X}$ is the association constant for ligand $X$ (when ligand $L$ is not bound to the macromolecule). 
Though, in general, equation 8 may seem difficult to be used in practical terms, an interesting and very useful linkage relationship can be obtained by calculating the derivative of the apparent association constant for ligand $L$ respect to the ligand $X$ concentration $[27,30]$ :

$\left(\frac{\partial \ln K_{a}^{a p p}}{\partial \ln [X]}\right)_{T, P, \ldots}=\sum_{j}^{m}\left(\frac{K_{j}^{\prime}[X]}{1+K_{j}^{\prime}[X]}-\frac{K_{j}[X]}{1+K_{j}[X]}\right)=\Delta n_{X}$

where $\Delta \mathrm{n}_{\mathrm{X}}$ is the net variation in the number of ligand $\mathrm{X}$ molecules bound to the macromolecule when ligand $L$ binds to the macromolecule. Therefore, if $\Delta n_{X}$ is equal to zero, ligand $L$ and ligand $X$ bind independently to the macromolecule, and the apparent binding parameters for ligand $\mathrm{L}$ will be independent of the ligand $\mathrm{X}$ concentration; otherwise, ligand $\mathrm{L}$ and ligand $\mathrm{X}$ bind cooperatively, and the apparent binding parameters for ligand $L$ will be dependent on the ligand $X$ concentration. Thus, performing titrations with ligand $L$ at different concentrations of ligand $X$ it is possible to estimate $\Delta \mathrm{n}_{\mathrm{X}}$ and assess such potential cooperativity between ligand $\mathrm{L}$ and ligand $\mathrm{X}$, and, in principle, it would be possible to estimate the intrinsic binding parameters by removing the contributions of ligand $X$ binding to the apparent binding parameters. It is important to stress that $\Delta n_{x}$ is not necessarily an integer number, since it is the difference in the occupancy of ligand $X$ between the L-bound macromolecule and the L-free macromolecule. Following the previous example regarding proton association/dissociation coupled to the binding of ligand $\mathrm{L}$ :

$\left(\frac{\partial \log K_{a}^{a p p}}{\partial p H}\right)_{T, P, \ldots}=-\Delta n_{H}$

which indicates that if the association constant for ligand $L$ depends on the $\mathrm{pH}$, the slope in the plot $\log \mathrm{K}_{\mathrm{a}}{ }^{\mathrm{app}}$ vs. $\mathrm{pH}$ is equal to the opposite of the net number of protons exchanged between the $M L$ complex and the bulk solution; if $\Delta n_{H}$ is positive, the formation of the complex $\mathrm{ML}$ is coupled to a net protonation of ionizable groups (positive cooperativity between ligand $L$ and protons), and if $\Delta n_{H}$ is negative, the formation of the complex $\mathrm{ML}$ is coupled to a net deprotonation of ionizable groups (negative cooperativity between ligand $L$ and protons). Thus, performing titrations at different $\mathrm{pH}$ 's it is possible to estimate $\Delta \mathrm{n}_{H}$. Another version of Equation 11 results in the following relationship [31]:

$\left(\frac{\partial \log K_{a}^{a p p}}{\text { aosmolality }}\right)_{T, P, \ldots}=-\frac{\Delta n_{W}}{55.6}$ 
which indicates that performing titrations under different osmotic stress conditions (e.g., different concentrations of osmolytes) it is possible to estimate the approximate number of water molecules, $\Delta \mathrm{n}_{\mathrm{W}}$, sequestered or released (mainly at the binding interface) upon formation of the ML complex.

It is then obvious that in general the observed binding parameters determined from a given titration under certain experimental conditions must be considered as apparent binding parameters, since, in principle, many additional equilibria (e.g., exchange of protons and other ions, binding of co-solutes, solvent release or sequestering, oligomerization/dissociation equilibrium, buffer de/ionization...) may be taking place coupled to the binding of ligand L. And even slight changes in the experimental conditions may result in considerable changes in the apparent binding parameters for the ligand. The contributions to the apparent binding parameters from certain extrinsic coupled equilibria should be eliminated (e.g., removing the influence of the buffer by using buffers with zero ionization enthalpy or extrapolating appropriately, removing the influence of ionic strength by extrapolating at zero ionic strength or at $1 \mathrm{M}$ ionic concentration), however, some coupled equilibria may be considered as inherent and essential events within the overall ligand binding process (e.g., protonation of a certain functional group in the macromolecule, oligomerization induced by ligand binding) and/or it might be difficult to dissect and remove their contributions to the overall apparent binding parameters. In any case, careful reporting of the experimental conditions and the characterization of reactants ( $\mathrm{M}$ and $\mathrm{L}$ ) is compulsory for avoiding misinterpretations and mistakes when considering the thermodynamic binding information for ligand optimization.

\subsection{The Gibbs energy change}

The Gibbs energy is the main thermodynamic potential in processes occurring at constant temperature and pressure, and it arises in a natural way when the second law of thermodynamics is applied to a closed system in thermal and mechanical equilibrium with its environment (at constant $T$ and $P$ ). The Gibbs energy change in a process is the maximum reversible non-expansion work that may be performed by a given closed system in thermal and mechanical equilibrium with its environment, and is related to the change in total entropy (system plus environment). For a process to 
occur spontaneously the Gibbs energy difference between the final and initial states must be negative; in particular, for binding processes the Gibbs energy change is the difference in chemical potential between the complex and the free species:

$\Delta G=\mu(M L)-\mu(M)-\mu(L)$

where $\mu$ is the chemical potential of a given type of molecule.

Because in any spontaneous binding process the Gibbs energy change is negative (the more negative $\Delta \mathrm{G}$, the higher the binding affinity), there is more interest in focusing on and discuss the thermodynamics of binding in terms of its partition into the enthalpic and the entropic contributions, which may show positive or negative changes and exhibit higher susceptibility to changes in intrinsic and extrinsic factors (e.g., structural/functional alterations in the binding partners, changes in experimental conditions) than the Gibbs energy [32], providing another layer of information on the binding process.

\subsection{The enthalpy change}

The enthalpy is a measure of the total energy in a system. At constant pressure, the enthalpy change in a system equals the energy exchanged with the environment through heat or non-expansion work; that is, the heat exchanged by the system a chemical reaction or by external heat transfer.

In binding process the enthalpy change reflects the net formation and disruption of many non-covalent interactions (hydrogen bonds, van der Waals and electrostatic interactions) between the two binding partners and the solvent, with the latter playing a key role establishing specific interactions and unspecific interactions. As indicated above, the overall enthalpy change (apparent or observed enthalpy of binding) can be split into the intrinsic enthalpy change plus the contributions from all possible coupled equilibria:

$\Delta H^{a p p}=\Delta H_{\text {int }}+\Delta H_{\text {coupled equilibria }}$

and, in a more specific way:

$\Delta H^{a p p}=\Delta H_{\text {int }}+\Delta H_{\text {desolv }}+\Delta H_{\text {conf }}+\Delta H_{\text {ex }}+\cdots$

where $\Delta \mathrm{H}_{\text {int }}$ is the intrinsic enthalpy (enthalpy change associated with the formation of non-covalent interactions between ligand and macromolecule), $\Delta \mathrm{H}_{\text {desolv }}$ is the desolvation enthalpy (enthalpy change associated with the disruption of non-covalent 
interactions between the binding partners and hydration water molecules), $\Delta \mathrm{H}_{\text {conf }}$ is the conformational enthalpy (enthalpy change associated with the conformational changes, in the ligand and the macromolecule, coupled to ligand binding; these conformational changes comprise large backbone rearrangements involving tertiary and secondary structure reorganization in the macromolecule, but also changes in flexibility of both the ligand and the side-chains of the macromolecule), and $\Delta H_{\mathrm{ex}}$ is the ligand-exchange enthalpy (enthalpy change associated with the exchange, association or dissociation, of other additional ligands upon ligand binding, such as ions, protons, small molecules...). Some of these additional contributions to the enthalpy may be regarded as an inherent part of the binding process (e.g., $\Delta \mathrm{H}_{\text {desolv }}, \Delta \mathrm{H}_{\text {conf }}$ ), while others are extrinsic contributions that, if possible, should be removed (e.g., $\Delta \mathrm{H}_{\mathrm{ex}}$ ).

Structurally different ligands will exhibit different contributions to the enthalpy change, making difficult the assessment and comparison of their binding thermodynamics based on the binding enthalpy (although, this also occurs with the Gibbs energy). Extensive experimental work may be required for quantifying individual enthalpic contributions. However, comparison of congeneric compounds obtained through bioisosteric substitutions may be considerably easier since those compounds may share common values for most of extrinsic contributions to the binding enthalpy (e.g., $\Delta \mathrm{H}_{\text {conf }}, \Delta \mathrm{H}_{\mathrm{ex}}$ ), and, therefore, differences observed between similar compounds may reflect specific changes in the contributions that can be engineered during the ligand optimization procedure. It is usual to group the intrinsic enthalpy and the desolvation enthalpy (and even the conformational enthalpy) into an "effective" binding enthalpy when approaching ligand optimization; being the other contributions important, they may be similar for the series of compounds or they may not be amenable to be engineered. Thus, the binding enthalpy can be considered a direct reflection of the interactions established between the binding partners to form the complex, the interactions broken with the solvent during desolvation (and the intramolecular interactions related to conformational changes).

Especial mention merits the coupling of proton exchange upon macromolecule-ligand complex. Because the experimental assay is performed with a buffered solution, the buffer molecule will be involved in any proton exchange in order to maintain constant the $\mathrm{pH}$. The apparent association constant and the apparent Gibbs energy of the ligand 
will not be affected by the ionization properties of the buffer as long as its $p K_{a}$ is close enough to the experimental $\mathrm{pH}$. But there will be a contribution to the binding enthalpy from the de/protonation of the buffer, which is an extrinsic contribution to the binding and could even dominate the apparent binding enthalpy $[26,33]$ :

$\Delta H^{\text {app }}=\Delta H_{0}+\Delta n_{H} \Delta H_{\text {buffer }}$

where $\Delta H_{\text {buffer }}$ is the ionization enthalpy of the buffer, and $\Delta H_{0}$ is the bufferindependent binding enthalpy (all other contributions). Thus, it is possible to remove the contribution from the buffer de/protonation by performing titrations with buffers with different ionization enthalpies. Very importantly, the factor $\Delta \mathrm{n}_{H}$ is the same as that appearing in Equation 12. Then, Equation 17 represents another method to determine the net number of protons exchanged by the macromolecule-ligand complex upon ligand binding, and the potential weakness is transformed into an advantage, since, in principle it is possible to determine the functional residues in the macromolecule and/or the ligand responsible for the proton exchange and their proton ionization properties (in particular, $\mathrm{pK}_{\mathrm{a}}$ values, which may be important regarding bioavailability of compounds) [34-37].

\subsection{The entropy change}

The entropy is a measure of the distribution of energy over the different energy levels and degrees of freedom in a given system. It is also considered a measure of the randomness or disorder within the system. As it occurs with the enthalpy change, the entropy change can be factorized into different contributions:

$-T \Delta S^{a p p}=-T \Delta S_{\text {desolv }}-T \Delta S_{\text {conf }}-T \Delta S_{\text {ex }}-T \Delta S_{r t}-T \Delta S_{v i b}-\cdots \ldots$

where $-\mathrm{T} \Delta \mathrm{S}_{\text {desolv }}$ is the desolvation entropy (entropy change associated with the disruption of non-covalent interactions between the binding partners and hydration water molecules), $-\mathrm{T} \Delta \mathrm{S}_{\text {conf }}$ is the conformational entropy (entropy change associated with the conformational changes, in the ligand and the macromolecule, coupled to ligand binding), $-\mathrm{T} \Delta \mathrm{S}_{\mathrm{ex}}$ is the ligand exchange entropy (entropy change associated with the exchange, association or dissociation, of other additional ligands upon ligand binding, such as ions, protons, small molecules...), $-\mathrm{T} \Delta \mathrm{S}_{\mathrm{rt}}$ is the roto-translational entropy (entropy change associated with the formation of a complex from two 
molecules with independent translational and rotational degrees of freedom), and $\mathrm{T} \Delta \mathrm{S}_{\mathrm{vib}}$ is the vibrational entropy (entropy change associated with vibrational mobility in covalent bonds in both binding partners).

Similar to the binding enthalpy, differences observed between congeneric compounds may reflect specific changes in the contributions that can be engineered during the ligand optimization procedure. It is usual to mainly focus on the desolvation entropy and the conformational entropy when approaching ligand optimization.

\subsection{The heat capacity change}

The heat capacity change is a measure of the ability to store thermal energy in a given system. It is also considered a measure of the fluctuations in the enthalpy of the system. As it occurs with the other parameters, the heat capacity change can be factorized into different contributions:

$\Delta C_{P}^{a p p}=\Delta C_{P, \text { desolv }}+\Delta C_{P, \text { conf }}+\Delta C_{P, \text { ex }}+\Delta C_{P, r t}+\Delta C_{P, v i b} \cdots$

where $\Delta C_{P, \text { desolv }}$ is the desolvation heat capacity, $\Delta C_{P, \text { conf }}$ is the conformational heat capacity, $\Delta C_{P, e x}$ is the ligand exchange heat capacity, $\Delta C_{P, r t}$ is the roto-translational heat capacity, and $\Delta \mathrm{C}_{\mathrm{P}, \mathrm{vib}}$ is the vibrational heat capacity (heat capacity associated with covalent bond vibrational modes in the interacting molecules).

Although the heat capacity of macromolecules is dominated by the vibrational contribution, the change in the heat capacity upon ligand binding is usually dominated by the desolvation contribution [38-40], with a significant but small contribution originated from electrostatic interactions [41]. This binding parameter is extremely useful for assessing conformational changes coupled to ligand binding or characterizing the role of water molecules incorporated into the binding interface. However, in this review we will focus on the Gibbs energy, the enthalpy and the entropy of ligand binding.

\section{Thermodynamics in Drug Discovery: Binding}

\subsection{The thermodynamic binding profile: Basics}

The measured binding parameters are apparent parameters containing contributions from any possible equilibrium coupled to ligand binding. Moreover, the thermodynamic binding parameters are very sensitive to changes in the interacting 
molecules (intrinsic factors) and changes in the experimental conditions (extrinsic factors). Controlling and describing in detail the experimental conditions is extremely important when reporting assay results. Additionally, it is also important to perform appropriate control assays, as well as to remove contributions from extrinsic factors when possible (e.g., contribution of the buffer de/protonation to the overall binding enthalpy). Dissecting the apparent binding parameters into individual contributions may be interesting, but for the purpose of using thermodynamic data in lead selection and optimization it may be not necessary. For example, the unfavorable rototranslational entropy is considerable, but it cannot be modulated and it will be the same for any 1:1 drug-target interaction.

The sensitivity of the apparent binding parameters to intrinsic and extrinsic factors is an indication of the wealth of valuable information on ligand binding (protonation, hydration, conformational changes, cooperativity...) that might be available with an adequately designed experimental strategy. Access to that information in early stages in the drug discovery process is critical towards selecting the best candidates and employing rational procedures in their optimization. For example, by performing structural or functional modifications in the ligand and assessing the effect observed in the apparent binding parameters it is possible to identify key functional groups in the interaction process (regarding binding affinity, binding enthalpy conformational change, cooperative effects...).

The formation of the drug-target complex recapitulates these contributions and can be conceptually split into three steps: 1) desolvation of the binding partners, 2) conformational adaptation of binding partners, and 3) formation of drug-target interactions.

The desolvation of binding partners involves the disruption of interactions between water molecules and polar groups from the target and the drug, and also water-water hydrogen bonds in molecules surrounding nonpolar groups. The desolvation is accompanied by an unfavorable enthalpy (a larger enthalpic penalty in polar groups, $\sim 8 \mathrm{kcal} / \mathrm{mol}$, compared to nonpolar groups, $\sim 0.7 \mathrm{kcal} / \mathrm{mol}$, due to disruption of interactions [42]), and a favorable entropy arising from the increased translational and vibrational mobility in the released water molecules. Interestingly, the desolvation entropy for polar groups is more favorable than that for nonpolar groups [42]. Of 
course, in the case some water molecules may become trapped into the binding interface, these will contribute with a favorable enthalpy and an unfavorable entropy. The conformational adaptation of the binding partners may range from loop reorganization in the macromolecule or rotatable bond restraint in the drug, to complete refolding of the macromolecule binding domain. While the enthalpy associated to the conformational change may be favorable or unfavorable, the entropy associated is usually unfavorable (although some individual contributions to the conformational entropy might be favorable [43], e.g., increase in flexibility in a certain region in the macromolecule upon ligand binding).

Finally, the formation of drug-target interactions relies on establishing hydrogen bonds and van der Waals and electrostatic interactions between drug and target. This is accompanied by a favorable enthalpy and an unfavorable entropy.

Summarizing, the overall binding entropy is dominated mainly by the large and favorable desolvation entropy associated with release of water molecules, and the unfavorable conformational entropy associated with the reduction in mobility of drug and target. On the other hand, the overall binding enthalpy is dominated by the large and unfavorable desolvation enthalpy (disruption of interactions with water) and the favorable interaction enthalpy (formation of drug-target interactions) (Figure 1).

\subsection{The thermodynamic binding profile: Interpretation}

From this basic description it is obvious that the enthalpy and entropy of binding reflect intermolecular interactions and phenomena of different nature: enthalpy mainly reflects specific (drug-target, drug-water and target-water) interactions, whereas entropy reflects unspecific interactions (hydrophobicity, shape complementarity, and conformational flexibility). Thus, the thermodynamic profile for a drug-target interaction reveals the mode of ligand interaction and the nature of the main intermolecular phenomena driving the interaction: 1) an enthalpically driven binding (unfavorable or small favorable binding entropy) indicates that the drug establishes better interactions with the target compared to those with water, and that the desolvation entropy hardly compensates the conformational entropy loss; and 2) an entropically driven binding (unfavorable or small favorable binding enthalpy) indicates unproductive interactions with the target that cannot compensate the 
disruption of interactions with water, and that the desolvation entropy largely compensates the conformational entropy loss. Therefore, in the absence of significant contributions from other coupled equilibria (conformational changes, protonation, etc.) or comparing leads affected by them in the same extent, scenario 1 may correspond to a flexible ligand with numerous polar groups establishing strong interactions with the target, whereas scenario 2 may correspond to a rigid ligand with numerous nonpolar groups establishing unspecific weak interactions with the target. Obviously, the two scenarios are completely different and the two ligands will differ in optimization potential, in binding selectivity and adaptability and drug-like quality. Thermodynamic profiles may be used to reveal different, alternative binding modes (outliers from the expected profile).

\subsection{The thermodynamic binding profile: Optimization}

From a thermodynamic point of view, the contributions to the Gibbs energy of binding that are more informative regarding the mode of interaction of the ligand, as well as more susceptible to be engineered during ligand optimization are (Figure 1): the effective binding enthalpy (intrinsic binding enthalpy and desolvation enthalpy), desolvation entropy, and conformational entropy. Drawing the attention to the binding entropy, the lead binding affinity can be improved by increasing the desolvation entropy (more hydrophobic by adding nonpolar groups; however, as commented before, the desolvation entropy of polar groups is more favorable [42]) and reducing the conformational entropy (pre-shaped to the binding site); additionally, the enthalpic penalty due to desolvation of nonpolar groups is reasonable small $[42,44]$. This has been the dominant optimization paradigm during the last decades, and, consequently, there is a tendency of new drug candidates to be excessively hydrophobic [45]; however, as explained below, this may have important drawbacks regarding solubility, bioavailability, and binding selectivity, diminishing the quality of the drug candidate. The question is: why has this happened? The answer is: because adding hydrophobic groups and structurally rigidifying the ligand molecule (entropic optimization) is much easier than adding polar groups for establishing distance- and orientation-dependent strong interactions with the target (enthalpic optimization). In principle, many combinations of binding enthalpy and entropy give rise to the same 
certain value of the binding affinity, however, an enthalpic ligand does not interact with the target in the same way as an entropic ligand does, even if they exhibit the same affinity: the former is attracted towards the target that provides an appropriate environment for satisfying energetic and functional constraints, whereas the latter just hides from water. Some drug-related properties, besides binding affinity, are directly linked to the enthalpy/entropy partition of the binding Gibbs energy. In addition, when optimizing leads already in the high affinity range, the possibility to optimize binding affinity with any combination of enthalpy and entropy is limited considering the constraints in the finite ensemble of chemical functionalities in small molecules, the different origin of enthalpic and entropic contributions, and the additional limitations imposed for maintaining or improving drug-like properties. Hence, when working at the high affinity range, it is desirable to avoid or minimize unfavorable contributions to the Gibbs energy and some degree of correlation between binding affinity and binding enthalpy arises.

As a conclusion, because the thermodynamic profile conditions the compound properties and the enthalpic optimization is difficult, starting from enthalpic hits or leads may seem advantageous and advisable; moreover, employing an enthalpic optimization strategy may be beneficial in early-medium stages in the drug discovery process because it will determine the drug quality of the compound, and entropic optimization can be performed in late-stage optimization [15]. The development of inhibitors against HIV-1 protease is an example of the expected benefits from using thermodynamics in drug design. The first HIV-1 protease inhibitors for clinical therapy were approved between 1995 and 1997. However, it took 12 years to develop secondgeneration inhibitors with improved binding affinity, binding selectivity, adaptability to drug-resistance associated mutations, and lower toxicity. One of the most striking conclusions from this study is the gradual, but dramatic, improvement in enthalpy: "first-in-class" inhibitors exhibit an unfavorable or small favorable enthalpy (entropically driven binding), while "best-in-class" inhibitors exhibit a large and favorable binding enthalpy (enthalpically driven binding) [46], a result also found for other targets like HMG-CoA reductase [18] and farnesyl pyrophosphate synthase [47]. Figure 2 shows the structures of the HIV-1 protease complexes for indinavir, KNI-764 and TMC-126. The binding Gibbs energies span a $3 \mathrm{kcal} / \mathrm{mol}$ range, but the binding 
enthalpy shows a $14 \mathrm{kcal} / \mathrm{mol}$ variation. Very importantly, binding enthalpy emerged as a major factor for affinity improvement (in addition to improvements in selectivity, adaptability, and side-effects), but it was not employed as a guiding criterion. What if binding enthalpy would have been integrated into the battery of decision-making tools in the drug discovery process? Other interesting conclusions can be extracted from the retrospective study on HIV-1 protease and other targets [48,49]: 1) the enthalpic character for the binding does not depend on the target; it is possible to develop enthalpically-driven and entropically-driven ligands for a given target; 2) there appear to be a certain optimal combination of enthalpy/entropy of binding; and 3) there is no correlation between the enthalpic character of a ligand and the structural/functional descriptors in the ligand. For example, the number of donors and acceptors of hydrogen bonds is not correlated with the enthalpic nature of their binding. Thus, the key point is not the number but the quality of the newly formed interactions between drug and target. Just adding polar groups to the ligand may result in an unfavorable binding enthalpy if they are not correctly positioned, because the enthalpic penalty from desolvation would not be compensated by sufficient enthalpic gain from new interactions. Therefore, unless a polar group establishes strong interactions with the target, it will contribute unfavorably or very low to the binding affinity, and it should be removed or modified during affinity optimization. This calls into question the utility of some cheminformatic filters, like the Lipinski's Rule of Five, in drug discovery and development.

\section{Thermodynamics in Drug Discovery: Drug-like Properties}

\subsection{LipE}

Among the many parameters employed as efficiency metrics to describe and rank ligand binding, lipophilic efficiency, LipE, is attracting much attention nowadays [50]:

$\operatorname{Lip} E=p K_{d}-\log P$

where $P$ is the octanol/water partition coefficient. Thus, LipE links binding affinity and hydrophobicity (or lipophilicity) in a single parameter and it is used in drug design and drug discovery to evaluate the quality of compounds and druglikeness. LipE quantifies the proportion of binding affinity that cannot be attributed to hydrophobicity (i.e., originated from polar groups). In practice, $\mathrm{pK}_{\mathrm{d}}$ can be substituted by pIC50 or pEC50, 
but, as commented above, IC50 and EC50 suffer from severe drawbacks. Similarly, logP can be substituted by $\log \mathrm{D}$ (considering neutral and ionized species), and in practice they can be substituted by their computational estimations ClogP or ClogD. On one hand, high potency is a desirable attribute in drug candidates. On the other hand, hydrophobicity is linked to reduced solubility, bioavailability, and binding selectivity, as well as increased toxicity, thus increasing the risk of ADMET-related attrition [51-54]. Increasing LipE results in improved binding affinity and ADMET properties $[55,56]$.

Theoretically, because: 1) polar groups contributing to the binding affinity also contribute to the binding enthalpy, and 2) polar groups exhibit low hydrophobicity, it is reasonable to expect a correlation between LipE and binding enthalpy. Interestingly, it has been shown experimentally that LipE correlates with binding enthalpy in many targets [57-59]. Thus, high LipE compounds exhibit the characteristic behavior associated with enthalpically driven binding (high selectivity, favorable ADMET properties), whereas low LipE compounds exhibit the characteristic behavior associated with entropically driven binding (low selectivity and impaired ADMET properties). That correlation may be founded on reasonable theoretical grounds [57], considering some assumptions (e.g., the binding entropy is dominated by the solvation entropy or the conformational entropy is similar for a given set of compounds); however, this connection has been questioned recently [22].

\subsection{Binding selectivity}

Binding selectivity is instrumental for avoiding drug binding to unwanted targets. Offtarget binding results in side-effects and toxicity. As indicated above, high hydrophobicity in leads may be linked to selectivity loss and high binding promiscuity. On the contrary, an enthalpic ligand will show considerable binding selectivity, because its binding is driven by polar, specific interactions [60]. Experimental examples have been found for the correlation between binding selectivity and binding enthalpy: compounds stabilizing the transthyretin tetramer [61], second-generation HIV-1 protease inhibitors [48], MMP12 inhibitors [62], and cannabinoid receptor ligands [63]. It has been found experimentally that, during the lead optimization process, the addition of a polar group establishing strong hydrogen bonds usually result in a small or negligible binding affinity improvement (large enthalpy gain is compensated by 
large entropy loss), an example of the enthalpy-entropy compensation phenomenon in drug discovery [64]. On the contrary, the addition of a nonpolar group very often results in a moderate binding affinity improvement (small enthalpy change is largely compensated by a large entropy gain). As indicated above, these two facts summarize the empirical foundations for the traditional lead optimization strategy based on the entropic optimization: use of nonpolar groups and conformational rigidification of ligand structures as main engineering tools. Non-polar functionalities are easier to engineer and result in moderate affinity improvements, but at the cost of diminishing the quality of the drug regarding solubility, bioavailability and selectivity. While polar functionalities are much more difficult to engineer (although their desolvation entropy is more favorable than that of nonpolar groups, there is a need to compensate the desolvation enthalpy penalty and overcome the enthalpy-entropy compensation), they increase the quality of the drug, being those functionalities the dominant driving force in second-generation or best-in-class drugs [46].

Enthalpy-entropy compensation is one of the main obstacles in drug discovery and development, and in the implementation of the enthalpic optimization of leads. The most difficult task is the introduction of polar groups (which ones? where?). After binding, polar groups may become: 1) exposed to the solvent and act as solubilizing elements of hydrophobic compounds; 2) desolvated, but not establishing strong interactions with the target; 3 ) desolvated establishing strong interactions with the target, but not contributing to binding affinity (due to enthalpy-entropy compensation); and 4) desolvated establishing strong interactions with the target and contributing to binding affinity (overcoming enthalpy-entropy compensation) [65]. Polar groups from category 2 should be eliminated immediately in the optimization process. Polar groups from category 4 must be directed towards structured, low mobility regions in the target, in order to avoid a conformational entropy loss upon binding and overcome the enthalpy-entropy barrier. Interestingly, polar groups from category 3 and 4 may contribute to binding selectivity, independently of their contribution to binding affinity [65]. The reasoning behind this fact relies on: 1) the large desolvation enthalpy penalty from polar groups; and 2) the specific differences in the location of key functional groups in the binding site of similar targets. Thus, an enthalpically optimized compound possesses carefully positioned polar groups, 
specifically mapping complementary groups in the target to achieve large favorable binding enthalpy. In a secondary target with a slightly distinct binding site, suboptimal match between certain complementary groups will result in unproductive desolvation and a less favorable, or even unfavorable, binding enthalpy, severely diminishing the binding affinity. On the other hand, an entropically optimized compound has fewer configurational constraints and a lower dependence from its environment (the compound is pushed towards the binding site by water). Kawasaki and Freire have delineated a strategy to improve binding selectivity through structural/functional modification of the lead: if binding selectivity is to be improved by increasing the binding affinity for the primary target more than that for secondary targets, nonpolar functionalities must be incorporated into the lead; on the contrary, if binding selectivity is to be improved by decreasing the binding affinity for the secondary targets more than that for the primary target, polar functionalities must be incorporated into the lead [65]. It is obvious that these two alternatives will modify the thermodynamic binding profile of the lead in a different fashion.

In general, selectivity does not require high affinity [23]. Binding affinity and binding selectivity are not correlated: binding affinity is related to the quality (strength, contribution to the overall Gibbs energy, as well as the partition into enthalpic and entropic terms) of polar interactions, while binding selectivity is related to the spatial configuration of polar interactions. Polar groups establishing good interactions in the binding interface will compensate their large desolvation enthalpic penalty with their intrinsic enthalpy and their desolvation entropy (high quality polar interactions), while polar groups establishing poor interactions in the binding interface will not compensate their large desolvation enthalpic penalty with their intrinsic enthalpy and their desolvation entropy (thus, not contributing to the binding or even decreasing the binding affinity). Therefore, a high affinity ligand may exhibit low binding selectivity, while a moderate affinity ligand may exhibit a high binding selectivity. In fact, given that the selectivity indexes for similar targets are usually not larger than 1000 , an extremely large binding affinity for the primary target may be detrimental for binding selectivity, because the binding affinity towards off-targets will still be considerable at the typical therapeutic dose of the drug [60]. Therefore, the best drug is not the compound with higher binding affinity, but the compound with sufficient binding 
affinity for eliciting the sought therapeutic effect, while exhibiting good pharmacokinetic and pharmacodynamic properties.

\subsection{Binding adaptability}

Binding adaptability is a concept apparently opposed to binding selectivity. Some targets require high target selectivity, while in other cases, because of natural/induced diversity or redundancy, adaptive drugs able to keep sufficient binding affinity for a set of homologous targets are required. For example, three of the most important adverse effects in HIV therapy based on protease inhibitors are: 1) side effects due to inhibition of secondary host targets, 2) the emergence of drug-resistant strains less susceptible to inhibitor action, and 3) the natural genetic diversity of HIV giving rise to coexisting multiple viral genotypes and subtypes. Then, adaptive drugs able to maintain enough binding affinity to distorted binding sites in the mutant or variant strains, but at the same time, able to maintain reduced binding affinity towards other host proteases are desirable. The proposed lead optimization strategy relies on the fact that mutations in the primary target occur at random only at certain residue locations, while key functional residues remain unchanged. Therefore, inhibitors must be engineered to establish strong interactions with conserved and more stable regions in the target and to find suboptimal complementarity in secondary targets providing binding selectivity, and to contain rotatable and asymmetric groups interacting with variable and less stable regions providing binding adaptability; entropic penalty associated with conformational flexibility in the ligand is compensated with the additional favorable binding enthalpy $[11,16,48,66,67]$.

\subsection{Allostery}

Ligand binding is very often coupled to considerable conformational changes. It is not that the binding triggers the conformational change, but, more appropriate, that the ligand binding shifts a pre-existing conformational equilibrium and changes the populations of conformational macrostates in a significant proportion. This is the molecular basis of allosteric control and allosteric phenomena in biomolecules: the modulation of a conformational equilibrium through ligand binding by stabilizing (populating) certain conformational states exhibiting specific biological activities. The 
conformational equilibrium will be reflected in the apparent thermodynamic parameters as one of the many possible contributions. In the case of remarkable conformational changes occurring simultaneously to ligand binding, the binding enthalpy and entropy might exhibit very large, similar in magnitude, opposing values (very favorable binding enthalpy and very unfavorable binding entropy) [68-70]. The thermodynamic binding profile can be used to discriminate between ligands promoting the conformational change and ligands not eliciting such conformational change, which would correspond to discriminate between activators and inhibitors if the conformational change is required for activity. An example of this strategy is the identification of small drugs able to bind to gp120 from HIV-1, but not eliciting the conformational change in gp120 mediated by CD4 and required for subsequent interaction with the chemokine receptor CCR5 or CXCR4 involved in viral entry inside the cell $[71,72]$.

\section{Thermodynamics in Drug Discovery: Caveats}

The previous section is full of optimistic statements about the application of thermodynamics in lead selection and optimization. However, extreme care should be taken when using thermodynamic binding profiles.

The binding process is a superposition of multiple microscale phenomena showing compensatory and cooperative effects, and binding enthalpy and entropy originate from multiplicity of intermolecular interactions [73]. Factorizing a thermodynamic binding profile into individual contributions is not trivial and rationalizing thermodynamic binding profiles may become a difficult task [74]. Careful reporting of experimental conditions in published work cannot be overemphasized.

Enthalpy-entropy compensation and cooperative energetic coupling between water molecules, functional groups in the ligand and intrinsic dynamics in the macromolecule may render quite difficult the interpretation of a given thermodynamic profile [75]. In particular, it has been shown that water molecules may considerably alter the thermodynamic binding profile, even if the effect on the Gibbs energy is small [76]. Thus, interactions expected to be dominated by the hydrophobic effect show a large, favorable binding enthalpy: polyproline recognition by $\mathrm{SH} 3$ domains [77], 
allophenylnorstatine-based inhibitors of the HIV-1 protease $[36,67]$, and inhibitors of aldose reductase [78].

Although enthalpy-entropy compensation, a formidable barrier to overcome in drug discovery, is a pervasive phenomenon in ligand binding [79], the correlation between enthalpy and entropy can be artificially enhanced by an incorrect experimental strategy where additional extrinsic equilibria are not taken into account, bad quality experimental data, and a defective data analysis procedure $[80,81]$.

In protein folding a good correlation between structural parameters (e.g., changes in solvent-accessible surface area) with energetic parameters has been established. However, this has been not the case in ligand binding, mainly due to the small size and wide structural diversity of ligands. Moreover, contrary to what happen in protein folding, the probability of establishing suboptimal interactions in ligand binding is high. Structural similarity in ligands (or in targets) does not necessarily result in similarity in binding energetics and allosteric effects, because intrinsic binding parameters may be differentially combined with contributions from coupled equilibria, water molecules, binding dynamics, or cooperativity between functional groups. Thus, the structural/functional determinants for binding affinity do not necessarily coincide with those required for eliciting cooperativity effects and allosteric responses: two similar ligands may exhibit different thermodynamic profiles and different allosteric and cooperativity effects with a given target [82-84], and a given ligand may exhibit different thermodynamic profiles and different allosteric and cooperativity effects with two homologous targets [85].

\section{Conclusions}

Biophysical techniques, in particular calorimetry, are important in the preclinical stage in drug discovery. They can be used for target characterization, screening validation, target engagement, and ligand binding analysis and optimization. In general, ligand binding is coupled to multiple equilibria (mainly, conformational, oligomerization, and ligand-exchange equilibria). The thermodynamic binding profile provides rich and valuable information on the ligand-target interaction, and ITC is the appropriate technique for dissecting the multiple contributions to the enthalpy and entropy of binding stemming from the different coupled equilibria. It is extremely important to 
always report the experimental conditions employed and the technical procedures employed for dissecting out the energetic contributions from the extrinsic factors regarding ligand binding, in order to be able to make appropriate use of the thermodynamic data when performing correlation analyses during quantitative energetics-structure-activity relationship (QESAR) studies.

Traditionally, lead optimization has been performed by making compounds more hydrophobic and pre-shaping to the target binding site. This entropic optimization results in poorer drug-like (ADMET) properties. The alternative consists of performing an enthalpic optimization engineering strong polar interactions from the earlier stages in drug discovery and combining, if necessary, with entropic optimization in the final stages. This has been proven to be the optimal strategy for maintaining or improving ADMET properties during binding affinity optimization. Potency, the drug dose needed for a certain physiological effect, is not only direct consequence of affinity, but also ADMET properties). Therefore, drug potency is related to both binding affinity and binding enthalpy, but binding selectivity and ADMET properties are mainly related to binding enthalpy.

We have reviewed the basis of ligand binding thermodynamics and the different contributions to the binding enthalpy and entropy. Then, we have discussed: 1) the consequences of implementing an enthalpic or entropic optimization of leads, 2) the empirical relation between binding enthalpy and drug-like properties and some optimization indexes, and 3) the rules for improving binding affinity while preserving or improving the quality of leads during optimization.

\section{Expert opinion}

How can we maximize likelihood of success and minimize risk in drug discovery? By integrating as much information as possible through orthogonal approaches: gathering biochemical, thermodynamic, kinetic, structural, and preclinical information alongside computational simulations. ITC is the gold-standard for characterizing biological interactions. Besides providing the "reduced" thermodynamic binding profile for a given drug-target interaction, ITC gives access to many energetic aspects (the "extended" thermodynamic binding profile), very often overlooked when attention is restricted to the Gibbs energy [86]. Additional equilibria coupled to ligand binding may 
trigger changes in the Gibbs energy of binding, but much larger changes in its enthalpic and entropic contributions. This may be seen as a weakness or as an advantageous feature allowing extracting valuable information on the ligand binding. Nevertheless, enthalpy and entropy of binding should not be used as direct end-points during optimization, but as a complementary tool for comparing ligand binding modes and identifying differential energetic outcomes between ligands (and the underlying molecular events and structural features).

In the past the thermodynamic binding profile has been employed retrospectively, together with structural information, as a guiding tool for lead optimization. Nowadays there is a quite strong foundation for using it prospectively in conjunction either with structural tools or within structure-activity relationship studies. Enthalpic ligand screenings can be routinely performed [87], and algorithms for implementing an enthalpic (or entropic) optimization are available [88]. Empirical rules for improving binding affinity, selectivity, and adaptability on an energetic basis have been established through experimental examples. However, the rather limited set of experimental systems employed to devise and formulate those rules has been appreciated as a strong limitation in their applicability. An extension to many other targets should be performed in order to test their validity, but also a closer look into those targets in which apparently they do not work would be convenient.

The comparison of thermodynamic binding profiles corresponding to different compounds is better performed on congeneric series of compounds containing bioisosteric substitutions. Thus, additional equilibria coupled to binding (and their contributions to the apparent binding parameters) are rather similar and the focus is placed on $\Delta \mathrm{H}_{\text {int }}, \Delta \mathrm{H}_{\text {desolv }},-\mathrm{T} \Delta \mathrm{S}_{\text {desolv, }}$ and $-\mathrm{T} \Delta \mathrm{S}_{\text {conf }}$.

Two key challenging phenomena must be solved in the short term in order to render thermodynamic binding profiles more informative and amenable to direct interpretation: energetic contribution of water molecules in the binding process, and cooperativity (non-additivity) of energetic contributions of ligand functional groups resulting in non-linear changes in the binding parameters. In the absence of structural information these two phenomena are quite difficult to unravel.

Enthalpic optimization through computational strategies is challenging because of the high sensitivity of binding parameters to intrinsic (structural and functional properties 
of interacting molecules) and extrinsic factors (experimental conditions and coupled equilibria), which exceeds the precision from current computational tools. Engineering polar interactions does not necessarily results in improved affinity, because of enthalpy-entropy compensation (compensation of desolvation enthalpy and conformational entropy penalties of polar groups with intrinsic enthalpy and desolvation entropic gains) and because of additional factors that are difficult to predict. Computational molecular design must be mainly directed to engineering polar groups, but, until computational tools for reliable prediction of the energetics of binding are available, the new thermodynamic binding profile after chemical modification of the lead must be obtained experimentally by ITC. Therefore, computational tools are very useful for molecular redesign and generation of sets of congeneric ligands, but we still must rely on experimental trial-and-error strategy. The comparison of the thermodynamic profile for a set of modified congeneric ligands regarding the parent unmodified lead will provide the information required for the next optimization steps.

Enthalpic optimization has been shown to be better than entropic optimization regarding the maintenance or improvement of quality of drug (ADMET properties). Some indexes to quantify ligand efficiency, employed in lead selection and optimization, involving the binding enthalpy have been defined: enthalpic efficiency ( $E E=\Delta H / N_{\text {pol }}, N_{\text {pol }}$ being the number of polar atoms in the ligand; or $E E=\Delta H / N_{h}, N_{h}$ being the number of non-hydrogen atoms in the ligand) and size-independent enthalpic efficiency (SIHE $=0.018 \times \Delta \mathrm{H} \times \mathrm{N}^{0.3}, \mathrm{~N}$ being the number of atoms in the ligand) $[14,89]$. However, lipophilic efficiency (LipE) has attracted much attention because it combines in a single number the enthalpic character of the ligand (key factor for binding affinity, selectivity and adaptability) and its hydrophobic nature (key factor for ADMET properties), and it has been proven to be associated with improved drug-like properties. The correlation between LipE and binding enthalpy, although not strictly rigorous, has been shown to occur experimentally for some targets and provides the basis for the enthalpic selection and optimization of leads in drug discovery.

The enthalpic optimization methodology is rather simple: 1) select enthalpically-driven leads; 2) analyze structural and functional variability in the macromolecule binding site; 3) focus initially on engineering polar interactions against conserved/stable 
subsites in the macromolecule binding site; 4) determine the new thermodynamic profile for modified ligands; 5) select new leads according to the improvement in binding enthalpy; and 6) engineer nonpolar interactions in late stages of ligand optimization. The correct application of the enthalpic optimization methodology requires: 1) following an appropriate experimental strategy (from the biophysical precharacterization of the binding partners to the selection of sets of experimental conditions) in order to identify extrinsic factors affecting the apparent binding parameters and eliminate their contributions, if possible, generating congeneric series of lead derivatives in a rational and smart way, and 2) obtaining high quality data and performing an adequate data analysis for minimizing experimental uncertainties and correlation between enthalpy and entropy. This statement, which in principle would be compulsory for any experimental technique, might represent a considerable barrier for the application of thermodynamic data in drug discovery.

Nobody can deny the initial expectations on the application of thermodynamic methods in drug discovery have not been met. But we may wonder whether the methodology is flawed and inefficient because it is just based on a few anecdotal observations and our understanding of binding thermodynamics is not sufficiently advanced, or the methodology may seem quite complex and cumbersome resulting in considerable reluctance from researchers to integrate it within drug discovery programs. Although the throughput of ITC is not high, instrumentation for satisfying the needs at medium scale, for secondary screenings or for sets of lead derivatives, is available, methodologies for increasing the quality and the throughput of experimental data have been developed (e.g., single-injection titration [90]), and empirical rules with reasonably solid foundations have been established to be applied on any potential target. Of course, it is not a straightforward task, but nobody said it was easy.

\section{Article highlights box}

- The thermodynamic binding profile (Gibbs energy, enthalpy and entropy of binding) provides a wealth of information on the lead-target interaction due to the high sensitivity of the apparent binding parameters to intrinsic and extrinsic factors.

- Thermodynamic ligand binding data seem to be underutilized in lead selection and optimization in drug discovery programs, and it may prove fundamental for the 
discovery of new drugs and the reduction of attrition rates when used in combination with biochemical, kinetic, structural, preclinical, and computational data.

- Traditionally lead optimization has been accomplished by optimizing binding entropy: increasing hydrophobicity and conformational rigidity in the ligand. This usually results in poor drug-like ADMET-related properties (e.g., solubility, bioavailability, toxicity).

- Binding enthalpy is related to binding affinity, selectivity, adaptability and drug-like properties. In particular, binding enthalpy seems to be correlated with lipophilic efficiency, a commonly used index for lead optimization rewarding binding affinity and penalizing hydrophobicity.

- Enthalpic optimization leads to better prioritization and optimization of leads, facilitating binding selectivity and adaptability, without compromising other druglike ADMET-related properties.

- The use of thermodynamic binding profiles requires carefully planned experimental strategy, high-quality experimental data, and good data analysis procedures.

\section{Declaration of interest}

This article was supported Spanish Ministerio de Economia y Competitividad [grants BFU2013-47064-P and BFU2016-78232-P to AVC], Fondo de Investigaciones Sanitarias from Instituto de Salud Carlos III [grant PI15/00663 to OA], Spanish Ministerio de Educacion, Cultura y Deporte [grant FPU13/3870 to RCG], Miguel Servet Program from Instituto de Salud Carlos III [grant CPII13/0017 to OA], Diputacion General de Aragon [grant B136/13 to RCG, Digestive Pathology Group B01 to OA and RCG, Protein Targets Group B89 to AVC], Centro de Investigacion Biomedica en Red en Enfermedades Hepaticas y Digestivas (CIBERehd), and Asociacion Española de Gastroenterologia (AEG).

The authors have no other relevant affiliations or financial involvement with any organization or entity with a financial interest in or financial conflict with the subject matter or materials discussed in the manuscript apart from those disclosed. 


\section{Bibliography}

Papers of special note have been highlighted as either of interest $(\bullet)$ or of considerable interest $(\bullet \bullet)$ to readers.

1. Copeland RA, Pompliano DL, Meek TD. Drug-target residence time and its implications for lead optimization. Nat Rev Drug Discov. 2006;5:730-9

- Introduction of the residence time as a new key optimization factor in lead optimization

2. Copeland RA. The drug-target residence time model: a 10-year retrospective. Nat Rev Drug Discov. 2016;15:87-95

3. Holdgate GA, Gill AL. Kinetic efficiency: the missing metric for enhancing compound quality? Drug Discov Today. 2011;16:910-3

4. Ladbury JE, Chowdhry BZ. Sensing the heat: the application of isothermal titration calorimetry to thermodynamic studies of biomolecular interactions. Chem Biol. 1996;3:791-801

5. Jelesarov I, Bosshard HR. Isothermal titration calorimetry and differential scanning calorimetry as complementary tools to investigate the energetics of biomolecular recognition. J Mol Recognit. 1999;12:3-18

6. Perozzo R, Folkers G, Scapozza L. Thermodynamics of protein-ligand interactions: history, presence, and future aspects. J Recept Signal Transduct Res. 2004;24:1-52

7. Holdgate GA, Ward WH. Measurements of binding thermodynamics in drug discovery. Drug Discov Today. 2005;10:1543-50

8. Velazquez-Campoy A, Todd MJ, Freire E. HIV-1 protease inhibitors: Enthalpic versus entropic optimization of the binding affinity. Biochemistry. 2000;39:2201-7

-• First study on the potential advantage in the enthalpic optimization of leads

9. Velazquez-Campoy A, Kiso Y, Freire E. The binding energetics of first and second generation HIV-1 protease inhibitors: implications for drug design. Arch Biochem Biophys. 2001;390:169-75

10. Velazquez-Campoy A, Luque I, Freire E. The application of thermodynamic methods in drug design. Thermochim Acta. 2001;380:217-27

11. Ohtaka H, Muzammil S, Schon A, et al. Thermodynamic rules for the design of high affinity HIV-1 protease inhibitors with adaptability to mutations and high selectivity towards unwanted targets. Int J Biochem Cell Biol. 2004;36:1787-99

12. Freire E. A thermodynamic guide to affinity optimization of drug candidates. In Proteomics and Protein-Protein Interactions: Biology, Chemistry, Bioinformatics, and Drug Design (ed. Waksman G), Springer, New York, 2005, p.291-307

13. Holdgate GA. Thermodynamics of binding interactions in the rational drug design process. Expert Opin Drug Discov. 2007;2:1103-14

14. Ladbury JE, Klebe G, Freire E. Adding calorimetric data to decision making in lead discovery: a hot tip. Nat Rev Drug Discov. 2010;9:23-7 
-• One of the first reviews on enthalpic optimization of leads, gathering thermodynamic data from different unrelated targets

15. Ferenczy GG, Keseru GM. Thermodynamics guided lead discovery and optimization. Drug Discov Today. 2010;15:919-32

16. Velazquez-Campoy A, Muzammil S, Ohtaka H, et al. Structural and thermodynamic basis of resistance to HIV-1 protease inhibition: implications for inhibitor design. Curr Drug Targets: Infect Disord. 2003 3:311-28

17. Nezami A, Kimura T, Hidaka K, et al. High-affinity inhibition of a family of Plasmodium falciparum proteases by a designed adaptive inhibitor. Biochemistry. 2003;42:8459-64

18. Carbonell T, Freire E. Biochemistry. Binding thermodynamics of statins to HMGCoA reductase. Biochemistry. 2005;44:11741-8

19. Klebe G. Applying thermodynamic profiling in lead finding and optimization. Nat Rev Drug Discov. 2015;14:95-110

- Comprehensive review of challenging aspects in the application of binding thermodynamics in drug discovery; in particular, cooperative effects, role of water molecules

20. Olsson TS, Ladbury JE, Pitt WR, et al. Extent of enthalpy-entropy compensation in protein-ligand interactions. Protein Sci. 2011;20:1607-18

21. Chodera JD, Mobley DL. Entropy-enthalpy compensation: role and ramifications in biomolecular ligand recognition and design. Annu Rev Biophys. 2013;42:121-42

22. Geschwindner S, Ulander J, Johansson P. Ligand binding thermodynamics in drug discovery: still a hot tip? J Med Chem. 2015;58:6321-35

-• Thought-provoking discussion on potential pit-falls and complexities in the use of binding thermodynamics in drug discovery

23. Chaires JB. Calorimetry and thermodynamics in drug design. Annu Rev Biophys. 2008;37:135-51

24. Garbett NC, Chaires JB. Thermodynamic studies for drug design and screening. Expert Opin Drug Discov. 2012;7:299-314

25. Renaud JP, Chung CW, Danielson UH, et al. Biophysics in drug discovery: impact, challenges and opportunities. Nat Rev Drug Discov. 2016;15:679-98

26. Eftink M, Biltonen R. Thermodynamics of interacting biological systems. In: Biological Calorimetry (ed. Beezer AE). Academic Press, London, 1980, pp.343-412

- Classic and up-to-date description of the coupling of some important molecular events linked to ligand binding and their influence on the thermodynamic binding profile

27. Wyman J, Gill SJ. Binding and Linkage: Functional Chemistry of Biological Macromolecules. University Science Books, Mill Valley, 1990

28. Vega $S$, Abian $O$, Velazquez-Campoy A. On the link between conformational changes, ligand binding and heat capacity. BBA - Gen Subjects. 2016;1860:868-878 
29. Velazquez-Campoy A, Goñi G, Peregrina JR, et al. Exact analysis of heterotropic interactions in proteins: characterization of cooperative ligand binding by isothermal titration calorimetry. Biophys J. 2006;91:1887-904

30. Vega $S$, Abian O, Velazquez-Campoy A. A unified framework based on the binding polynomial for characterizing biological systems by isothermal titration calorimetry. Methods. 2015;76:99-115

31. Parsegian, VA, Rand RP, Rau DC. Macromolecules and water: probing with osmotic stress. Methods Enzymol. 1995;259:43-94

32. Hinz HJ. Thermodynamics of protein-ligand interactions: calorimetric approaches. Annu Rev Biophys Bioeng. 1983;12:285-317

33. Hinz HJ, Shiao DDF, Sturtevant JM. Calorimetric investigation of inhibitor binding to rabbit muscle aldolase. Biochemistry. 2971;10:1347-52

34. Baker BM, Murphy KP. Evaluation of linked protonation effects in protein binding reactions using isothermal titration calorimetry. Biophys J. 1996;71:2049-55

35. Xie D, Gulnik S, Collins L, et al. Dissection of the $\mathrm{pH}$ dependence of inhibitor binding energetics for an aspartic protease: direct measurement of the protonation states of the catalytic aspartic acid residues. Biochemistry. 1997;36:16166-72

36. Velazquez-Campoy A, Luque I, Todd MJ, et al. Thermodynamic dissection of the binding energetics of KNI-272, a potent HIV-1 protease inhibitor. Protein Sci. 2000;9:1801-9

37. Linkuviene $V$, Matuliene J, Juozapaitiene $V$, et al. Intrinsic thermodynamics of inhibitor binding to human carbonic anhydrase IX. BBA - Gen Subjects. 2016;1860:708-18

38. Gomez J, Hilser VJ, Xie D, et al. The heat capacity of proteins. Proteins. $1995 ; 22: 404-12$

39. Prabhu NV, Sharp KA. Heat capacity of proteins. Annu Rev Phys Chem. 2005;56:521-48

40. Syme NR, Dennis C, Philips SEV, et al. Origin of heat capacity changes in a "nonclassical" hydrophobic interaction. ChemBioChem. 2007;8:1509-11

41. Gallagher K, Sharp K. Electrostatic contributions to heat capacity changes of DNAligand binding. Biophys J. 1998;75:769-76

42. Cabani S, Gianni P, Mollica V, et al. Group contributions to the thermodynamic properties of non-ionic organic solutes in dilute aqueous solution. J Solution Chem. 1981;10:563-95

43. Wand, AJ. The dark energy of proteins comes to light: conformational entropy and its role in protein function revealed by NMR relaxation. Curr Opin Struct Biol. 2013;23:75-81

44. Martin SF, Clements JH. Correlating structure and energetics in protein-ligand interactions: paradigms and paradoxes. Annu Rev Biochem. 2013;82:267-93 
45. Keseru GM, Makara GM. The influence of lead discovery strategies on the properties of drug candidates. Nat Rev Drug Discov. 2009;8(3):203-12

- Discusses how high-throughput screening programs and hit-to-lead optimization practices condition drug-like properties in leads

46. Freire E. Do enthalpy and entropy distinguish first in class from best in class? Drug Discov Today. 2008;13:869-874

- Discusses the benefits of including the binding enthalpy as an additional guiding criterion in drug discovery, presenting successful experimental examples in overcoming the enthalpy-entropy compensation barrier

47. Kawasaki Y, Sekiguchi M, Kawasaki M, et al. Thermodynamic evaluation of the binding of bisphosphonates to human farnesyl pyrophosphate synthase. Chem Pharm Bull (Tokyo). 2014;62:77-83

48. Ohtaka H, Freire E. Adaptive inhibitors of the HIV-1 protease. Prog Biophys Mol Biol. 2005;88:193-208

49. Ruben AJ, Kiso Y, Freire E. Overcoming roadblocks in lead optimization: A thermodynamic perspective. Chem Biol Drug Des. 2006;67:2-4

50. Freeman-Cook KD, Hoffman RL, Johnson TW. Lipophilic efficiency: the most important efficiency metric in medicinal. Future Med Chem. 2013;5:113-5

51. Hughes JD, Blagg J, Price DA, et al. Physiochemical drug properties associated with in vivo toxicological outcomes. Bioorg Med Chem Lett. 2008;18:4872-5

52. Waring MJ. Lipophilicity in drug discovery. Expert Opin Drug Discov. 2010;5:235-48

- Discusses how lipophilicity influences the quality of leads, by determining preclinical ADMET and the likelihood of success in development

53. Arnott JA, Planey, SL. The influence of lipophilicity in drug discovery and design. Expert Opin Drug Discov. 2012;7:863-75

54. Tarcsay A, Keseru GM. Contributions of molecular properties to drug promiscuity. J Med Chem. 2013;56:1789-95

55. Gleeson MP, Hersey A, Montanari D, et al. Probing the links between in vitro potency, ADMET and physicochemical parameters. Nat Rev Drug Discov. 2011;10:197-208

56. Tarcsay A, Nyiri K, Keseru GM. Impact of lipophilic efficiency on compound quality. J Med Chem. 2012;55:1252-60

57. Shultz MD. The thermodynamic basis for the use of lipophilic efficiency (LipE) in enthalpic optimizations. Bioorg Med Chem Lett. 2013;23:5992-6000

- Provides the pseudo-empirical foundation for the correlation between binding enthalpy and lipophilic efficiency, as well as experimental data substantiating such correlation

58. Hopkins AL, Keseru GM, Leeson PD, et al. The role of ligand efficiency metrics in drug discovery. Nat Rev Drug Discov. 2014;13:105-21 
59. Freire $\mathrm{E}$. The binding thermodynamics of drug candidates. In Thermodynamics and Kinetics of Drug Binding (ed. Keseru GM, Swinney DC), Wiley-VCH Verlag GmbH, 2015, pp.3-13

60. Tarcsay A, Keseru GM. Is there a link between selectivity and binding thermodynamics profiles? Drug Discov Today. 2015;20:86-94

- Discusses the relation between entropically-driven and enthalpically-driven binding with binding selectivity using experimental data from different unrelated targets

61. lakovleva I, Brannstroom K, Nilsson L, et al. Enthalpic forces correlate with the selectivity of transthyretin-stabilizing ligands in human plasma. J Med Chem. 2015;58:6507-515

62. Bertini I, Calderone V, Fragai $M$, et al. Exploring the subtleties of drug-receptor interactions: the case of matrix metalloproteinases. J Am Chem Soc. 2007;129:2466-75

63. Merighi S, Simioni C, Gessi S, et al. Binding thermodynamics at the human cannabinoid CB1 and CB2 receptors. Biochem Pharmacol. 2009;79:471-7

64. Lafont V, Armstrong AA, Ohtaka H, et al. Compensating enthalpic and entropic changes hinder binding affinity optimization. Chem Biol Drug Des. 2007;69:413-22

65. Kawasaki Y, Freire E. Finding a better path to drug selectivity. Drug Discov Today. 2011;16:985-90

-• Discusses how ligand binding selectivity can be engineered by adding polar or non-polar functionalities, and the consequences on the thermodynamic binding profile

66. Velazquez-Campoy A, Freire E. Incorporating target heterogeneity in drug design. J Cell Biochem. 2001;84(S37):82-8

- Discusses three main heterogeneity sources in target binding sites (genetic diversity, drug-induced resistance mutations, and binding site dynamics) and the development of adaptive ligands

67. Vega S, Kang LW, Velazquez-Campoy A, et al. A structural and thermodynamic escape mechanism from a drug resistant mutation of the HIV-1 protease. Proteins. 2004;55:594-602

- Detailed experimental example of ligand binding adaptability based on improved binding enthalpy and engineered conformational flexibility in the ligand

68. Kwong PD, Doyle ML, Casper DJ, et al. HIV-1 evades antibody-mediated neutralization through conformational masking of receptor-binding sites. Nature. 2002;420:678-682

69. Courter JR, Madani N, Sodroski J, et al. Structure-based design, synthesis and validation of CD4-mimetic small molecule inhibitors of HIV-1 entry: conversion of a viral entry agonist to an antagonist. Acc Chem Res. 2014;47:1228-37 
70. Edink E, Rucktooa P, Retra K, et al. Fragment growing induces conformational changes in acetylcholine-binding protein: a structural and thermodynamic analysis. J Am Chem Soc. 2011;133:5363-71

71. Schon A, Madani N, Klein JC, et al. Thermodynamics of binding of a low-molecularweight CD4 mimetic to HIV-1 gp120. Biochemistry. 2006;45:10973-80

72. Schon A, Madani N, Smith III AB, et al. Some binding-related drug properties are dependent on thermodynamic signature. Chem Biol Drug Des. 2011;77:161-5 - Ligand optimization based on the binding thermodynamic profile aimed at discriminating ligands depending on whether they elicit allosteric conformational changes in the target

73. Bissantz C, Kuhn B, Stahl M. A medicinal chemist's guide to molecular interactions. J Med Chem. 2010;53:5061-84

74. Talhout R, Villa A, Mark AE, et al. Understanding binding affinity: a combined isothermal titration calorimetry/molecular dynamics study of the binding of a series of hydrophobically modified benzamidinium chloride inhibitors to trypsin. J Am Chem Soc. 2003;125:10570-9

75. Fenley AT, Muddana HS, Gilson MK. Entropy-enthalpy transduction caused by conformational shifts can obscure the forces driving protein-ligand binding. Proc Natl Acad Sci USA. 2012;109:20006-11

76. Breiten B, Lockett MR, Sherman W, et al. Water networks contribute to enthalpy/entropy compensation in protein-ligand binding. J Am Chem Soc. 2013;135:15579-84

77. Zafra-Ruano A, Luque I. Interfacial water molecules in SH3 interactions: getting the full picture on polyproline recognition by protein-protein interaction domains. FEBS Lett. 2012;586:2619-30

78. Steuber $H$, Heine A, Klebe $G$. Structural and thermodynamic study on aldose reductase: nitro-substituted inhibitors with strong enthalpic binding contribution. J Mol Biol. 2007;368:618-38

79. Eftink MR, Anusiem AC, Biltonen RL. Enthalpy-entropy compensation and heat capacity changes for protein-ligand interactions: general thermodynamic models and data for the binding of nucleotides to ribonuclease A. Biochemistry. 1983;22:3884-96

80. Tellinghuisen J, Chodera JD. Systematic errors in isothermal titration calorimetry: concentrations and baselines. Anal Biochem. 2011;414:297-9

81. Tellinghuisen J. Isothermal titration calorimetry at very low c. Anal Biochem. 2008;373:395-7

82. Williams R, Holyoak T, McDonald G, et al. Differentiating a ligand's chemical requirements for allosteric interactions from those for protein binding. Phenylalanine inhibition of pyruvate kinase. Biochemistry. 2006;45:5421-9 
83. Ruiz-Ramos A, Velazquez-Campoy A, Grande-Garcia A, et al. Structure and functional characterization of human aspartate transcarbamoylase, the target of the anti-tumoral drug PALA. Structure. 2016;24:1081-94

84. Liu Y, Schon A, Freire E. Optimization of CD4/gp120 inhibitors by thermodynamicguided alanine-scanning mutagenesis. Chem Biol Drug Des. 2013;81:72-8

-. Target hotspots contributing to affinity do not necessarily contribute to allostery

85. Jobichen C, Fernandis AZ, Velazquez-Campoy A, et al. Identification and characterization of the lipid-binding property of $\mathrm{GrIR}$, a locus of enterocyte effacement regulator. Biochem J. 2009;420:191-9

86. Ladbury JE, Williams MA. The extended interface: measuring non-local effects in biomolecular interactions. Curr Opin Struct Biol. 2004;14:562-9

- Review on different phenomena coupled to ligand binding, in particular the contribution from ionic exchange, hydration changes and folding

87. Schon A, Freire E. Enthalpy screen of drug candidates. Anal Biochem. 2016;513:1-6

88. Freire E. A thermodynamic approach to the affinity optimization of drug candidates. Chem Biol Drug Des. 2009;74:468-72

89. Ferenczy GG, Keseru GM. Enthalpic efficiency of ligand binding. J Chem Inf Model. 2010;50:1536-41

90. Markova N, Hallen D. The development of a continuous isothermal titration calorimetric method for equilibrium studies. Anal Biochem. 2004;331:77-88

\section{Figure legends}

Figure 1. Main enthalpic (green) and entropic (red) contributions to the Gibbs energy of binding (blue). The intrinsic enthalpy and the desolvation enthalpy are always favorable and unfavorable, respectively; however, other contributions such as the conformational enthalpy and the ligand-exchange enthalpy may favor or oppose binding (represented in attenuated color). On the other hand, the desolvation entropy is always favorable, while the conformational entropy and the roto-translational entropy are always unfavorable; however, other contributions such as the ligandexchange entropy may favor or oppose binding (represented in attenuated color). The sum of all contributions gives rise to the Gibbs energy of binding.

Figure 2. Crystallographic structures of HIV-1 protease bound to indinavir (PDB code 2bpx), KNI-764 (PDB code 1msm), and TMC-126 (PDB code 214u). The thermodynamic binding profile for the three inhibitors under identical experimental conditions is 
shown. The binding of the three inhibitors is accompanied by a similar conformational change in the target, since the RMSD between two given structures is not larger than $0.7 \AA$. The large differences in binding enthalpy (corrected for proton exchange processes) between the three ligands cannot be justified considering the polar groups in each molecule and the hydrogen bonds established between drug and target.

Indinavir contains 9 polar groups: 3 groups are establishing 6 hydrogen bonds with the target, 4 groups are establishing 4 hydrogen bonds with water molecules, and 2 groups are not involved in hydrogen bonds formation.

KNI-764 contains 9 polar groups: 4 groups are establishing 7 hydrogen bonds with the target, 4 groups are establishing 4 hydrogen bonds with water molecules, and 1 group is not involved in hydrogen bonds formation.

TMC-126 contains 11 polar groups: 5 groups are establishing 7 hydrogen bonds with the target, 2 groups are establishing 2 hydrogen bonds with water molecules, and 4 groups are not involved in hydrogen bonds formation. 


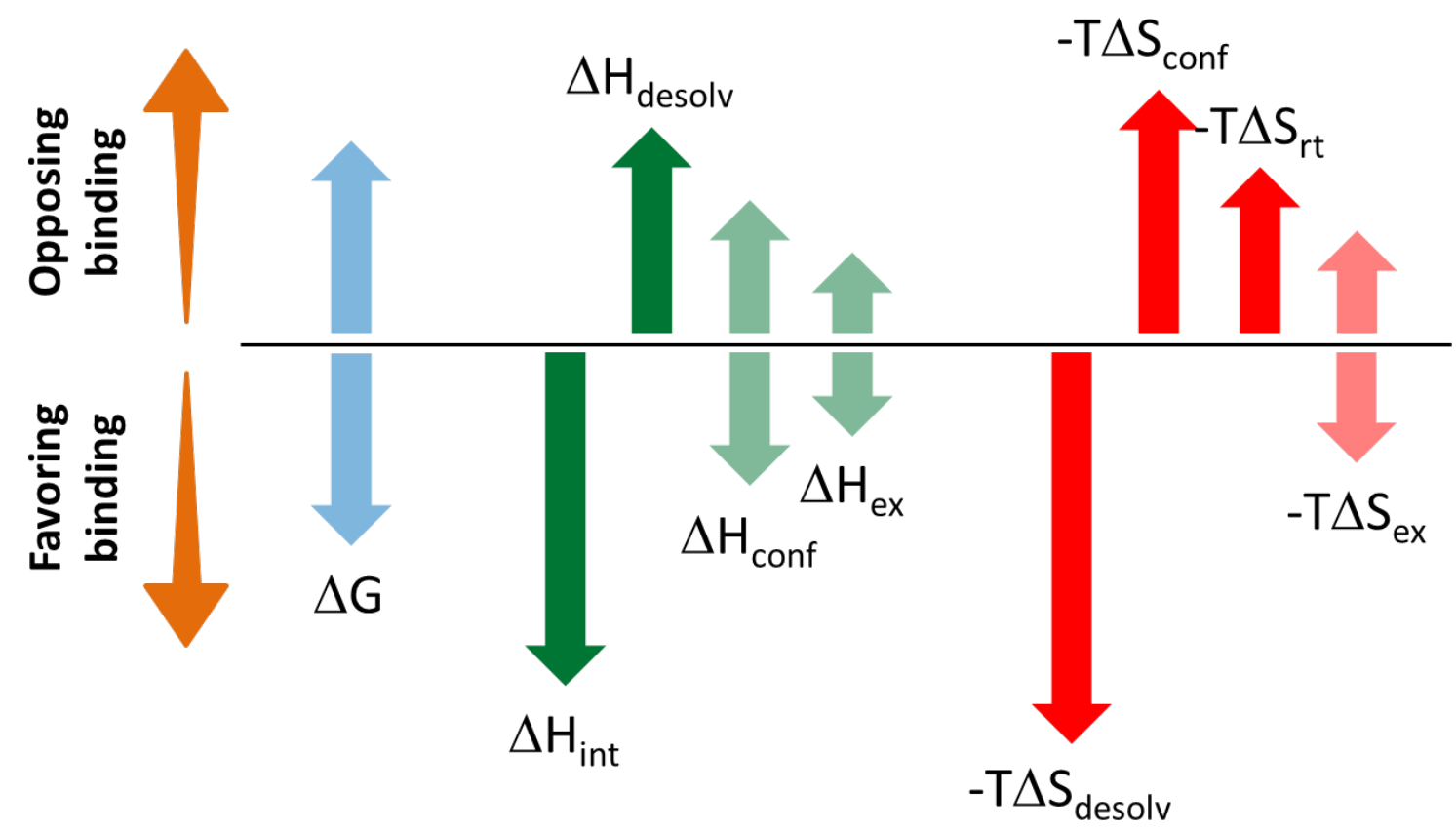

Figure 1
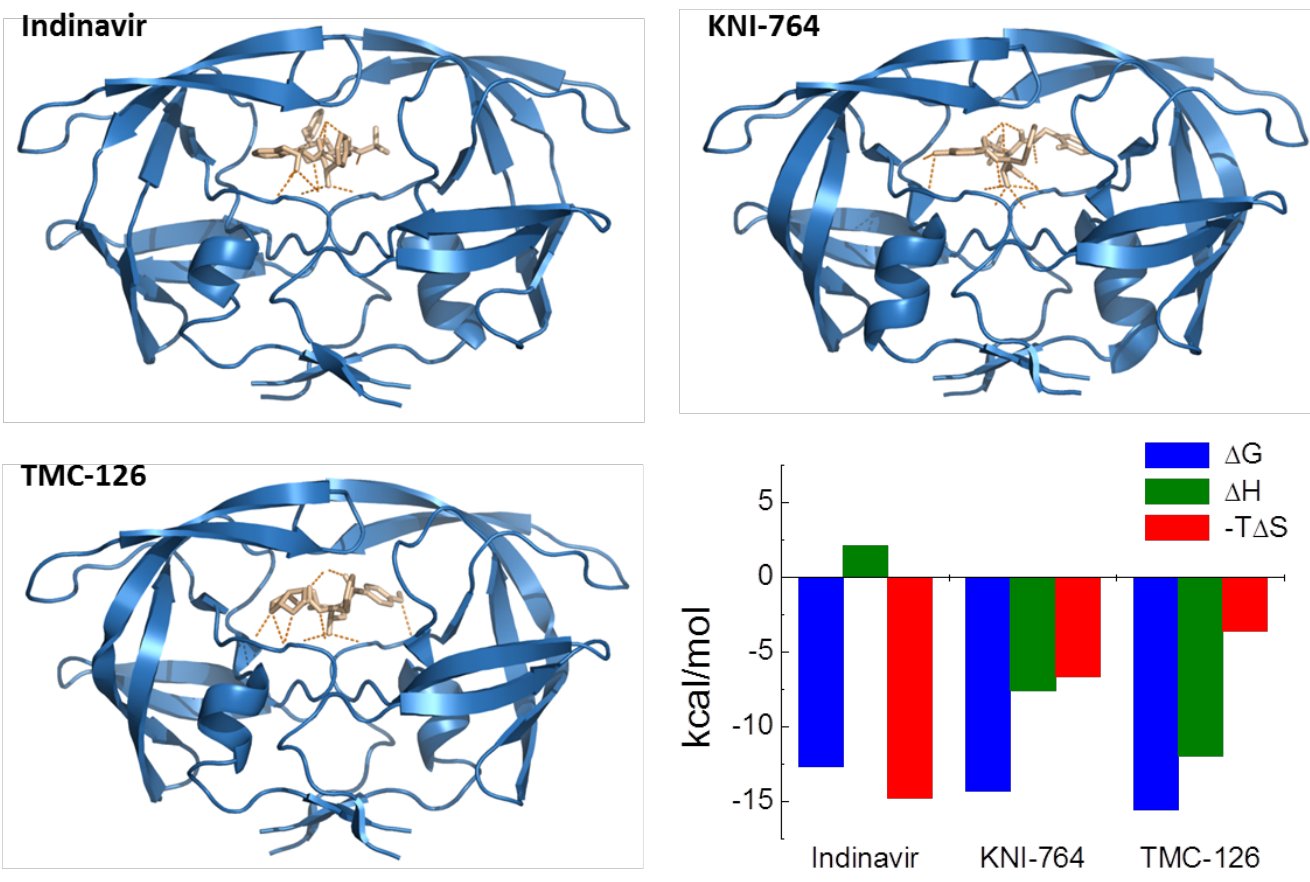

Figure 2 